\title{
Combining rails and anchors with laser forcing for selective manipulation within 2D droplet arrays
}

\author{
Etienne Fradet, ${ }^{a}$ Craig McDougall, ${ }^{b}$ Paul Abbyad, ${ }^{c}$ Rémi Dangla, ${ }^{a}$ David McGloin ${ }^{b}$ and Charles N. Baroud ${ }^{a}$
}

We demonstrate the combination of a rails and anchors microfluidic system with laser forcing to enable the creation of highly controllable 2D droplet arrays. Water droplets residing in an oil phase can be pinned to anchor holes made in the base of a microfluidic channel, enabling the creation of arrays by the appropriate patterning of such holes. The introduction of laser forcing, via laser induced thermocapillary forces to anchored droplets, enables the selective extraction of particular droplets from an array. We also demonstrate that such anchor arrays can be filled with multiple, in our case two, droplets each and that if such droplets have different chemical contents, the application of a laser at their interface triggers their merging and a chemical reaction to take place. Finally by adding guiding rails within the microfluidic structure we can selectively fill large scale arrays with monodisperse droplets with significant control over their contents. In this way we make a droplet array filled with 96 droplets containing different concentrations of fluorescent microparticles.

\section{Introduction}

Performing a set of independent reactions in a two-dimensional (2D) array format is a standard approach for implementing a large number of parallel assays. This is the case for instance in multiwell plates, DNA or protein chips, and many other genomic technologies. Droplet microfluidic techniques have aimed to replicate such a $2 \mathrm{D}$ format in recent years, since the ability to keep a drop stationary allows the long term observation of its contents, in addition to providing combinatorial measurements on a single image. Patterning a 2D area with different droplets is straightforward when using surface microfluidic manipulations, e.g. through electro-wetting ${ }^{1}$ or surface acoustic waves. ${ }^{2}$ More classical drop deposition techniques have also been used to produce arrays of distinct droplets for combinatorial enzymatic studies ${ }^{3}$ or for polymerase chain reaction (PCR). ${ }^{4}$

Producing arrays of droplets in microchannels has proved more challenging, in part due to the standard methods for forming drops: both $\mathrm{T}$-junctions and flow-focusing devices rely on the presence of a strong flow of the carrier fluid, ${ }^{5}$ so that holding the drops in an array requires a method to stop their motion against the outer flow. This has been achieved through

${ }^{a}$ Laboratoire d'Hydrodynamique (LadHyX) and Department of Mechanics, Ecole Polytechnique, CNRS, 91128, Palaiseau, France. E-mail: baroud@ladhyx.polytechnique.fr

${ }^{b}$ Electronic Engineering and Physics Division, University of Dundee, Nethergate, Dundee, DD1 4HN, UK

${ }^{c}$ Laboratoire d'Optique et Biosciences (LOB), Ecole Polytechnique, INSERM U696, CNRS, 91128 Palaiseau, France innovative designs of the microfluidic geometries and operating protocols, leading to different methods to array drops: quasi-2D arrays were formed by winding a linear path in the plane of the microchannel. Drops flowed in series in this microchannel and were blocked at particular locations, for example by having side pockets in the channel, ${ }^{6,7}$ by flowing the drops into parallel deadend microchannels, ${ }^{8}$ or into parallel corrugated channels. ${ }^{9}$ These devices however suffer from strong droplet interactions since the drops are tightly confined into linear motion by the channel walls, which can make their operation difficult. Some authors reduced these interactions by increasing locally the channel width and depth. ${ }^{10}$ Real 2D arrays, in which droplet interactions are weak, were formed by placing obstacles in a wide microchannel ${ }^{11}$ and the obstacles could be designed to hold one, two or more drops. ${ }^{12,13}$

More recently, Abbyad et al. ${ }^{14}$ demonstrated the manipulation of drops in 2D by using rails and anchors, which correspond to grooves that are etched on the surface of the microchannel. In this approach, drops are squeezed by the top and bottom boundaries of the microchannel, which increases their surface energy. By partially entering into the grooves, the drop reduces its surface energy. This creates a gradient of surface energy at the edge of the grooves, which leads to an attractive force pulling it into the region with larger depth. This was used to guide drops along linear paths (a rail) or to hold them stationary in an anchor. ${ }^{14}$

The current challenge is therefore to pattern a 2D area with a heterogeneous droplet population, either by selectively placing a drop at the desired location, selectively extracting a drop, or selectively initiating a chemical reaction. To date, none of the methods for arraying drops allow for such controlled operations. 
Instead, random filling is used in most cases and there exists no mechanism for selectively extracting a particular drop. While random distribution of drops can yield useful information in the case of two droplet species, ${ }^{13}$ it quickly becomes limiting when there are many species to be studied simultaneously. Here we demonstrate how the controlled filling, extraction, or reactions can be performed by combining the rails and anchors approach with the selective manipulation through laser-induced local heating: ${ }^{15-17}$ The rails and anchors provide a passive, robust and simple 2D guidance and trapping of drops, while the mobile laser spot adds an intelligent and versatile selectivity.

After the materials and methods section (Section 2), we turn to the physical ingredients that determine the different forces in Section 3. This is followed by demonstrations of drop extraction in Section 4. Filling an array with a controlled drop population is then demonstrated by showing how to build an array of drops which displays a gradient of concentration (Section 5). Finally, we demonstrate how to trigger local reactions on demand (Section 6).

\section{Materials and methods}

\section{Microfluidic device fabrication}

All experiments were conducted in PDMS microchannels (Dow Corning Sylgard 184) sealed onto glass slides by plasma bonding. The fabrication procedure relies on dry film photoresist soft lithography techniques ${ }^{\mathbf{1 8}}$ which enable rapid prototyping of multi-level structures. The multilayer masters were etched in stacks of Eternal Laminar E8013 and Eternal Laminar E8020 negative films (of thickness $35 \pm 2 \mu \mathrm{m}$ and $50 \pm 2 \mu \mathrm{m}$ respectively) depending on the desired thickness of the main channel and patterns.

The successive steps were the following: (i) photoresist layers were successively laminated onto a clean glass slide using a PEAK Photo Laminator (PS320) at a temperature $T=100{ }^{\circ} \mathrm{C}$ until the desired height $h$ of the main channel was reached; (ii) The photoresist stack was exposed to UV (Hamamatsu Lightningcure LC8) through a photomask of the base channel (comprising a test section, droplet generation devices and entrance and exit channels); (iii) Additional photoresist layers were laminated on top of the exposed stack until the desired depth $p$ of the patterns (anchor holes and/or rails) was reached; (iv) The stack of photoresist films was exposed again to UV, through the second photomask featuring only the patterns to be added onto the base channel. Finally, the full structure was developed by immersion in an aqueous bath of carbonate potassium at $1 \%$ mass concentration.

Furthermore, in order to render the internal channel surface hydrophobic, a surface treatment was applied: A dilute solution of $1 \mathrm{H}, 1 \mathrm{H}, 2 \mathrm{H}, 2 \mathrm{H}$-perfluorodecyltrichlorosilane (Sigma-Aldrich) in FC40 oil (3M Fluorinert) $(20 \mu \mathrm{L}$ in $1 \mathrm{~mL}$ of FC40) was flowed through the microchannel for approximately $5 \mathrm{~min}$. The channel was then rinsed with pure FC40 to remove the residue chemicals remaining in the bulk.

\section{Chip design and operation}

The common architecture of the microfluidic chip was a base channel with a blank 'test section' onto which rail and anchor patterns were etched. The base channel consisted of one (Sections 4-5) or two (Section 6) droplet generation devices discharging into a wide main test section. The system then emptied into a single outlet channel. The height of the base channel $h$ was 100 $\mu \mathrm{m}$ everywhere. Channels upstream of the test section had widths ranging from 100 to $200 \mu \mathrm{m}$. The test section was a rectangle of dimensions $10 \times 3 \mathrm{~mm}$ (Sections 4 and 6 ) or $20 \times 4.5 \mathrm{~mm}$ (Section 5).

Various patterns of rails and anchors could then be added onto the base test section depending on the desired applications. Section 4 relied on a regular square array of small circular holes of diameter $d=50 \mu \mathrm{m}$ and depth $p=35 \mu \mathrm{m}$. For Section 6, only two rows of holes with a diameter $d=200 \mu \mathrm{m}$ were used. The pattern designed for Section 5 was a complex combination of rails, ranging in width from $50 \mu \mathrm{m}$ to $200 \mu \mathrm{m}$, combined with square anchors of $120 \mu \mathrm{m}$ a side.

Throughout the study, the continuous phase was FC40 oil (3M Fluorinert) of viscosity $\mu=4.1 \mathrm{cP}$ containing fluorinated surfactants for both emulsion stabilization and improvement of the wetting conditions. Experiments described in Sections 4 and 5 used a Krytox (Dupont) ammonium salt, ${ }^{19}$ at $0.5 \%$ mass concentration, while those in Section 6 used a PEG-based surfactant at $0.01 \%$ mass concentration. ${ }^{19}$ Two inlets of oil were required: one at a flow rate $Q_{\mathrm{o}}$ for the droplet generation and one at a flow rate $Q_{\mathrm{e}}$ to adjust droplet entrainment in the test section independently of the droplet generation.

Different aqueous solutions were used as the dispersed phase and were injected at a flow rate $Q_{\mathrm{w}}$ : Section 4 uses pure water droplets; in Section 5 a solution of water containing fluorescent beads (Invitrogen green fluorescent beads, $1 \mu \mathrm{m}$ ) at a volume fraction of $0.4 \%$. In Section 6 , the two aqueous solutions consisted of a solution of $\mathrm{FeCl}_{3}$ at $0.27 \mathrm{M}$ and a solution of $\mathrm{KSCN}$ at $0.8 \mathrm{M}$. The interfacial tension $\gamma$ between a pure water drop and the FC40 solution was measured to be $\sim 20 \mathrm{mN} \mathrm{m}^{-1}$. We observed however that the contents of the drops, such as the chemicals of Section 6 or the fluorescent beads, could have an impact and reduce this value in some cases.

A system of computer controlled syringe pumps (Cetoni neMESYS) was used to control the different flow rates $\left(Q_{\mathrm{w}}, Q_{\mathrm{o}}\right.$ and $Q_{\mathrm{e}}$ ) in real time.

\section{Optical setup and laser operation}

The optical arrangement employed for droplet manipulation makes use of a $1480 \mathrm{~nm}$ continuous wave infrared laser source (Fitel Furukawa FOL1424) and a commercial inverted microscope system (Nikon TE2000) equipped with epifluorescent illumination (Exfo X-cite 6210C). A pair of galvanometric mirrors (Cambridge Technologies $6210 \mathrm{H}$ ) permitted beam positioning in the microchannels to be controlled by a mouse click using in-house Labview programs. Following the galvanometric mirror, the laser beam was both expanded appropriately (to overfill the back aperture of the microscope objective) and relayed to the back aperture of the objective by a $4 \mathrm{f}$ conjugate lens system and an appropriate dichroic mirror (OCTAX). The laser power was measured to be $200 \mathrm{~mW}$ in the focal plane of the microscope objective. The described system provided an approximately diffraction limited spot at the focal plane whose position was readily computer controlled by the user. A camera 
was used to image the field of view via a sideport and record monochrome video at typical frame rates of 60 frames per second (Photron Fastcam 1024 PCI). Alternatively, a digital SLR camera (Nikon D70) was used to capture color images via the front camera port of the microscope. Whilst the laser spot wavelength was outside the range of both cameras, the spot location was visible as a dark shadow on the computer screen.

\section{Physical ingredients}

Guiding and trapping drops in our devices are based on manipulating three forces of different physical origins, as sketched in Fig. 1: The force due to the surface patterning $F_{\gamma}$, the entrainment force due to the flow of the outer fluid $F_{\mathrm{d}}$, and the force due to the laser heating $F_{1}$. Below we discuss the main attributes of each of these forces.

\section{Force due to surface patterning}

The surface energy of a droplet can be written as $E_{\gamma}=\gamma A$, where $\gamma$ is the interfacial energy and $A$ the surface area of the droplet. While $\gamma$ can be considered as nearly constant over the course of an experiment, $A$ can vary significantly as the droplet changes its shape. An unconfined drop will take a spherical shape in order to minimize its surface area while the confinement can be used to force the drop to take a flattened "pancake" shape of height nearly equal to the microchannel height $h$. In the plane of the microchannel, one can describe the drop by its radius $R$; in the case when $R \gg h$, the main contribution to the surface area is given by the top and bottom boundaries of the droplet and we can write $A \simeq 2 \pi R^{2}$, with a small correction due to the area around the drop in the vertical direction.

When the channel height is locally modified, for instance by etching a groove into one of the surfaces, the drop enters into the groove. This always leads to a reduction of the total surface area of the drop, ${ }^{20}$ thus reducing its surface energy. Removing the drop from the region of low surface energy therefore requires a force $F_{\gamma}$, whose magnitude is given by the gradient of the energy and always pointing towards the energy minimum. Dangla et al. ${ }^{20}$ estimate this anchoring force as $\gamma \Delta A / d$, where $\Delta A$ is the difference in surface area between the drop over the groove and away from it, and $d$ is a characteristic length scale over which

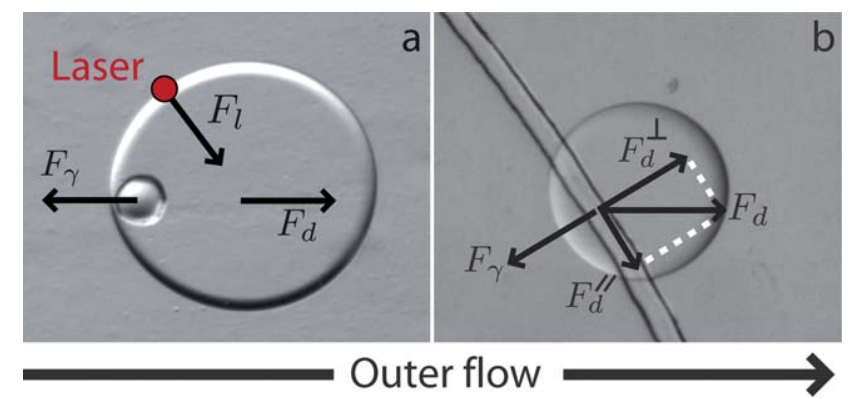

Fig. 1 Forces acting on a drop in the presence of surface patterning: The anchoring force due to the surface patterning $F_{\gamma}$, the drag force due to the flow of the outer fluid $F_{\mathrm{d}}$, and the force due to the laser heating $F_{1}$. (a) The case of an anchor. (b) In the case of a rail, the unbalanced force due to the drag parallel to the rail $F_{\mathrm{d}}^{\prime \prime}$ leads to motion along this direction. the energy changes. They measure forces in the range of 100-600 $\mathrm{nN}$ for similar geometries to the ones presented here.

For a circular groove, a geometric calculation shows that $F_{\gamma}$ does not depend on the drop size in the case when $R \gg h$. This is not the case however in the case of a rail, where $F_{\gamma}$ scales linearly with the drop radius $R$.

\section{Force due to the outer flow}

The flow of the outer fluid applies a drag force $F_{\mathrm{d}}$ on the droplet through hydrodynamic drag. This force is always directed in the direction of motion of the outer fluid. Its magnitude scales as $F_{\mathrm{d}} \sim \mu_{\mathrm{o}} U R^{2} / h$, where $\mu_{\mathrm{o}}$ is the outer fluid viscosity and $U$ is the velocity of the outer fluid. $F_{\mathrm{d}}$ therefore increases with the flow velocity and depends strongly on the size of the droplet.

For given drop and channel geometries, we can measure a critical velocity beyond which $F_{\mathrm{d}}>F_{\gamma}$ at which point the drop cannot be held anymore. For velocities below this critical velocity, a drop in an anchor is deformed from its circular shape (Fig. 1(a)). Conversely, a drop that is in a rail is pushed along the rail due to the existence of a component in the tangent direction $F_{\mathrm{d}}^{\prime \prime}$ of the drag force (Fig. 1(b)). Once the critical velocity is reached, drops cannot be held anymore and they are de-pinned by the outer flow. ${ }^{14}$ An important regime concerns velocities that are slightly below the critical velocity. In this regime, the anchor strength is sufficient to hold one droplet stationary but not two, since the net drag force experienced by the touching pair increases while the anchoring force remains constant. Abbyad et al. ${ }^{14}$ identified a range of velocities where drops enter into a 'buffering' mode, in which an incoming drop replaces the drop that was previously anchored. This buffering regime will be used below to determine the occupation of anchor sites in the experiments.

\section{Force due to the laser heating}

The final force $F_{1}$ that we must consider is due to the localized heating by a focused laser. ${ }^{21}$ When the water-oil interface is heated locally, variations in surface tension lead to the creation of a flow along the interface, which in turn leads to flow inside and outside the droplet. ${ }^{16}$ The net effect on the droplet is a "pushing" force, by which the laser heating forces the droplet away from the laser position. This has been used in one-dimensional (1D) microchannels to sort or buffer drops, or change the order in which they flow. ${ }^{17}$ The magnitude of $F_{1}$ was measured ${ }^{21}$ in confined microchannels to be in the range of $100-300 \mathrm{nN}$. Verneuil et al. also observed that the contributions to the force are dominated by the flows along the thin films between the drop and the lateral channel walls, which implied that the force depended on the drop size in that case. ${ }^{21}$ Finally, the direction of the force due to the laser heating is determined by the direction of the thermo-capillary flows. In the case studied by Verneuil et al. ${ }^{21}$ the presence of surfactants in the continuous phase implied that laser produced a repulsive force, by inducing anomalous thermo-capillarity. There are however no measurements of the magnitude or size dependence of $F_{1}$ in the absence of lateral walls. 


\section{Extracting a drop from an array of holes}

The force balance described above can now be used to selectively remove a drop from an anchor site, by adding the laser-induced force to the drag force from the fluid flow. An example implementation is shown in Fig. 2 and in ESI movie S1. $\dagger$ In this experiment, drops are initially formed in a flow-focusing geometry and flowed into the test section, which is patterned with a square lattice of holes. Some drops become anchored at the hole positions, filling the lattice in a random fashion. The water flow is then stopped and the oil flow rate is held constant at $Q_{\mathrm{o}}=$ $2 \mu \mathrm{L} \mathrm{min}{ }^{-1}$.

Once the lattice is filled (Fig. 2(a)), the laser focus is selectively positioned within the test section. If the laser is focused inside the droplet, it can remove it from the anchor. This allows the user to select a position on the computer screen and to remove the corresponding drop at that particular position. The oil flow rate then transports the drop away from the array and out of the microchannel (Fig. 2(b)). Fig. 2(c) shows an ' $X$ ' pattern formed using this method.

This device can be operated in two different regimes. In the current regime, small anchors and a weak oil flow rate are used, therefore requiring a high laser power $\left(P_{\text {laser }}=200 \mathrm{~mW}\right)$ to remove the drops. Alternatively, lower laser powers can be used by increasing the flow rate to work closer to the critical anchoring velocity. In that case however, extracted drops interact with their neighbours and can lead to a buffering mode, in which drops downstream of the extracted drop also get unpinned.

\section{Selectively filling an array}

Here, the ability to sort and subsequently fill a two-dimensional array with droplets of a uniform size is presented. The methodology is again based upon the combination of creative chip design, which combines rails and anchors of different strengths, and highly localised laser induced forcing for derailing the drops. By superimposing anchors onto rails, droplets may be guided directly to the anchor sites where they become trapped on these 'storage rails'. The presented method involves first selectively filling these storage rails and then allowing the droplets to assemble into the desired array by entering into a buffering mode through an increase of the carrier oil flow rate.

To construct an array of droplets, we use a chip test section composed of 6 rails, each of which is periodically superimposed with 16 anchor sites, producing a 96 drop array. The rail sorting portion of this test section is shown in Fig. 3(a) which demonstrates active switching of droplets between rails, achieved by employing laser-induced forces as described above. In the

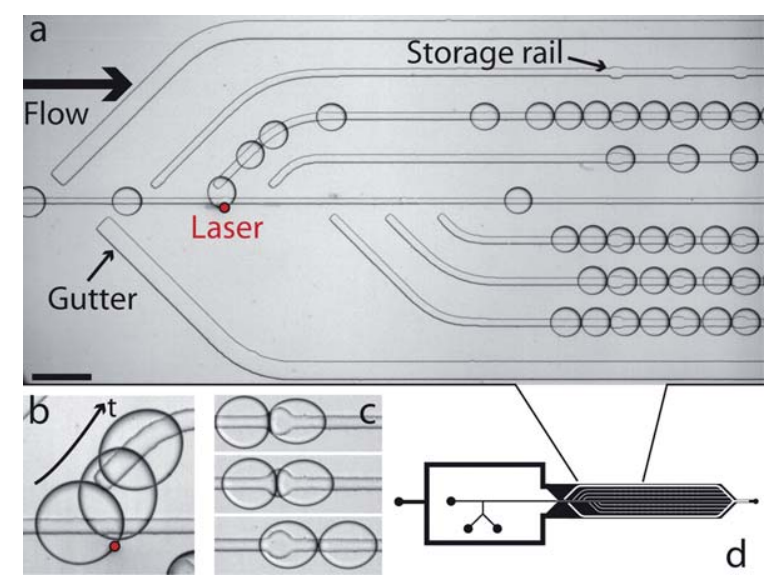

Fig. 3 (a) Test region $(20 \times 4.5 \mathrm{~mm}, h=100 \mu \mathrm{m})$ for building an array of droplets. All rails are $50 \mu \mathrm{m}$ deep. The central default rail is $50 \mu \mathrm{m}$ wide. The side gutter rails used to filter out large droplets are $200 \mu \mathrm{m}$ wide. The 6 storage rails are $75 \mu \mathrm{m}$ wide and the anchor sites superimposed on these rails are $120 \mu \mathrm{m}$ squares. The image shows the fifth rail being filled (see movie S2 for full sequence $\dagger$ ). The scale bar is $600 \mu \mathrm{m}$. (b) Superposition of three images showing a drop leaving the default rail into a storage rail. (c) Once the storage rails are filled the entrainment flow rate $Q_{\mathrm{e}}$ is increased to initiate a buffering mode whereby the droplets self arrange into an array of one droplet trapped at each anchor site. (d) A schematic of the complete chip design illustrates the droplet formation portions of the chip relative to the test region.

absence of the laser, droplets are guided along the central rail to the exit of the chip by default. The selective derailing operation is demonstrated in Fig. 3(b) and ESI movie S2. $\dagger$ To fill the array, rails are filled in sequence, row by row, beginning with the rightmost junction to the left-most junction. Any order can be used however, even working on a drop-by-drop basis.

Selective filling of the array, in addition to anchoring the drops in place, depends upon several key design features which are highly sensitive to droplet size. Consequently, the device geometry is constructed specifically for a given droplet size. For this experiment the chips (shown in Fig. 3(d)) are designed for droplets of a $150 \mu \mathrm{m}$ radius.

The first of these features is designed to ensure that all droplets placed on the array are of the same size. Such steps are necessary, as droplet monodispersity is not guaranteed for transient stages of droplet production, nor for all flow rates. Droplets are only placed in the array when the laser is used to direct them there. Furthermore, droplets below the size of interest cannot be derailed from the central rail, due to the limit on the maximum deflection achievable with the employed laser power. Small

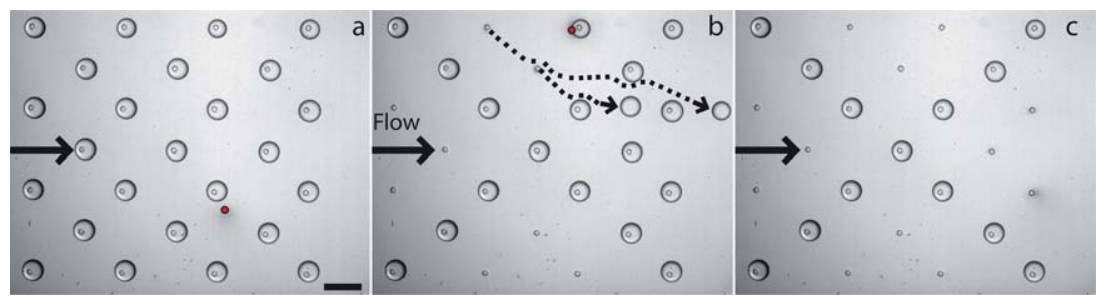

Fig. 2 Selectively extracting drops. (a) The array of holes ( $d=50 \mu \mathrm{m}$ and $p=35 \mu \mathrm{m})$ is initially filled with water drops. (b) The laser is focused on the water-oil interface, pushing the drops out of the anchor. (c) An X is patterned with the remaining droplets. Scale bar is $400 \mu \mathrm{m}$. 
droplets are therefore guided out of the chip and never reach the storage rails. To ensure droplets of a size larger than those under study are also eliminated, two side gutters are placed upstream and redirect large droplets along the side of the test section. Therefore, large droplets are prevented from entering the array portion of the chip as the gutter rails provide an energetically favorable alternative route. These features ensure that only droplets of the desired size are available for active laser forcing onto the array rails.

The remaining features are concerned with filling the storage rails. Once a droplet passes the two side gutters, it enters the rail switching area and is available for sorting into the storage rails containing square anchor sites. As can be seen in Fig. 3(b), the storage rails have been designed to be wider than the central guide rail and there is a gap between the storage rails and the central default rail. This intentional gap is to avoid junction sites, where the local width reaches a maximum value. This leads to junctions behaving as anchor sites from which drops cannot be removed. Upon deflection by the laser, the droplet sees the wider side rail as an energetically favorable route and leaves the default rail as illustrated in both Fig. 3(b) and ESI movie S2. $\dagger$

Once sorted onto the storage rails, the droplet encounters anchor sites and becomes trapped. The dimensions of the anchors have been chosen for the given droplet radii so that they may hold one droplet parked stationary against the mean flow. However, the anchor site is intentionally too weak to hold multiple droplets stationary. Consequently, under the correct external flow conditions the droplets will enter into a buffering regime. It is noteworthy that whilst the storage rails are being filled with droplets, the apparent channel cross section available for the oil to flow through decreases due to the stationary droplets. Such a reduction causes an increase in the velocity that must be taken into account when filling the anchor sites, to prevent all the droplets from being swept out of the array. Therefore, the entrainment oil flow rate $Q_{\mathrm{e}}$ is reduced from 40 to $20 \mu \mathrm{L} \mathrm{min}{ }^{-1}$ during the filling period as the storage rails become populated, while the small oil $\left(Q_{\mathrm{o}}\right)$ and water $\left(Q_{\mathrm{w}}\right)$ flow rates are maintained at a steady 0.5 and $0.18 \mu \mathrm{L} \mathrm{min}{ }^{-1}$ respectively. This ensures that all rails are completely filled with monodisperse droplets during the filling procedure. Once this procedure is completed, the entrainment flow rate $Q_{\mathrm{e}}$ is increased slowly to 70 $\mu \mathrm{L} \mathrm{min}{ }^{-1}$ to initiate the desired droplet buffering regime (Fig. 3 (c)). Under these conditions arrays of 96 droplets may be constructed in a controlled manner at a rate of approximately $1 \mathrm{~min}$ per rail for droplet sorting and a further minute for droplet buffering into a complete array.

Whilst maintaining the droplet size for a given chip design, this methodology may be extended to droplets of varying composition. An example of such a controlled filling appears in Fig. 4, where an array of 96 anchored droplets with an increasing concentration of fluorescent beads is shown. In the chip used here, a mixer was positioned before the T-junction used for droplet formation, allowing the composition of the droplets to be altered in time. To modify the composition of the droplets, a stream of pure water is mixed with a concentrated bead solution in pure water $(0.4 \%$ by volume). For the various rails of the array, the ratio of pure water to bead solution is altered by varying the relative flow rates whilst maintaining an overall flow rate of $0.18 \mu \mathrm{L} \mathrm{min}{ }^{-1}$ for the mixture. This ensures that the

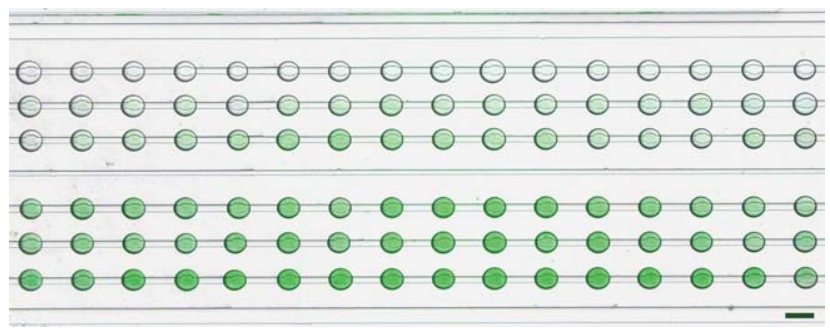

Fig. 4 A superposition of bright-field and fluorescent images of a 96 anchor array. The droplet composition has been altered from pure water to a solution of water and green fluorescent beads to create a gradient. The droplet volume is $7 \mathrm{~nL}$ each. The scale bar is $300 \mu \mathrm{m}$.

droplets are of uniform size. For the top rail, droplets are composed entirely of pure water and the flow rate of the bead solution is set to zero. For each row, moving down the array, the flow rate of the bead solution is increased in $0.005 \mu \mathrm{L} \mathrm{min}{ }^{-1}$ increments whilst the water rate is decreased by the same amount.

The filling protocol begins with the top rail, which is filled with droplets containing pure water. The laser is then removed, causing all droplets to follow the default central path to the drain. The droplet composition is then altered to the concentration required for the next rail and allowed to reach a steady state before filling of that rail commences. To do so we translate the laser to the correct position for the new rail and direct droplets into the storage rail. Once the rail is filled with droplets, the laser is again removed and the droplet composition altered. The remaining storage rails are filled in this stepwise manner, changing the droplet formation specifically for each rail and using the laser to position the droplets onto the rails.

\section{Controlled initiation of chemical reactions at anchor sites}

Our final demonstration of the power of the technique is to carry out the loading of two droplets into the anchor sites and then use laser induced merging of droplets to enable controlled chemical reactions within selected droplet pairs. This can be done at a location predefined by the anchor position and it indicates the potential of this method as an analysis and assaying tool.

We make use of an array of anchors in the chip test section. A key point here is that the anchor holes are designed to be twice as wide as the droplet diameters, so that each can accommodate two droplets. In this situation, where the holes are larger than the droplet, an outer flow rate of $80 \mu \mathrm{L} \mathrm{min}^{-1}$ removes one drop per trap while in the experiment presented in Section 4, an outer flow rate of $10 \mu \mathrm{L} \mathrm{min}{ }^{-1}$ detaches all the drops.

To initiate a chemical reaction we must load droplets with different contents into each hole site. We choose droplets with $\mathrm{FeCl}_{3}$ and $\mathrm{KSCN}$, which react to form a colored liquid from two colorless ones. We begin by generating one species of droplets from the top flow focusing junction and fill the anchor sites

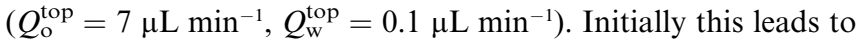
two droplets occupying each anchor site, since the holes are twice as big as the droplets (Fig. 5(a + b) and supplementary movie

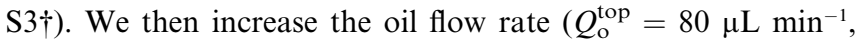
$Q_{\mathrm{w}}^{\text {top }}=0 \mu \mathrm{L} \mathrm{min}^{-1}$ ) to wash out one of each pair, thus leaving one 


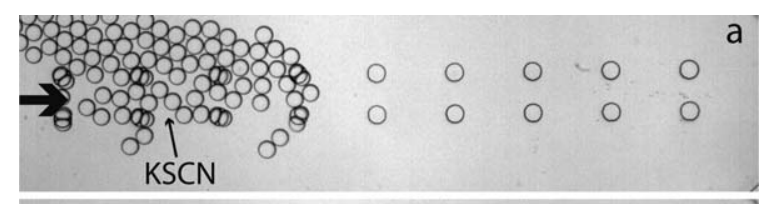

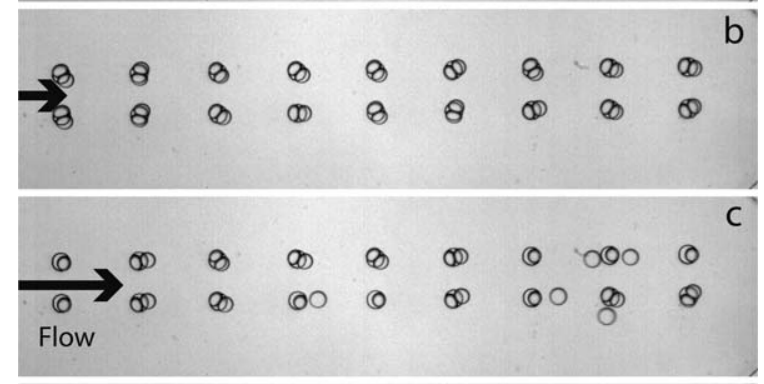

$\underset{0}{0}\left[\begin{array}{llllllll}0 & 0 & 0 & 0 & 0 & 0 & 0 & 0 \\ 0 & 0 & 0 & 0 & 0 & 0 & 0\end{array}\right.$

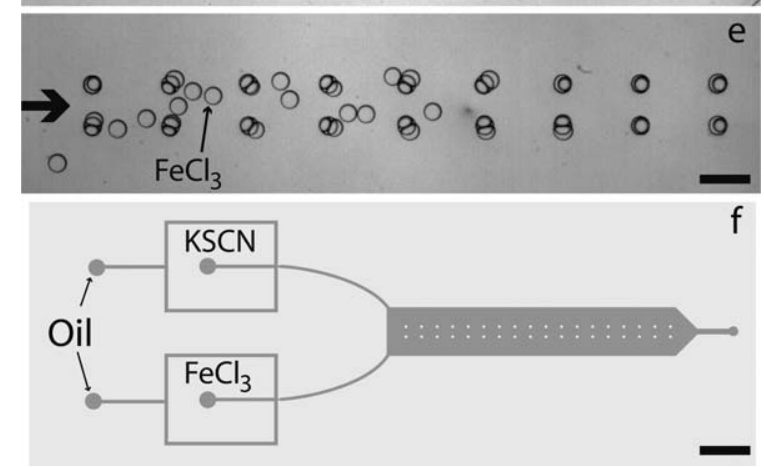

Fig. $5(a+b)$ Filling first species into anchor holes. $(c+d)$ Emptying one droplet from each trap to leave one drop per anchor site. (e) Bringing in the second species droplets to anchor holes. The scale bar is $400 \mu \mathrm{m}$. (f) Schematic of channel used, illustrating dual inlets for the two different chemical species. The test section (containing the anchors) is $100 \mu \mathrm{m}$ high, $1 \mathrm{~cm}$ long and $3 \mathrm{~mm}$ wide. The anchor holes have a height of $50 \mu \mathrm{m}$ and a diameter of $200 \mu \mathrm{m}$. The scale bar is $3 \mathrm{~mm}$.

droplet per site $($ Fig. 5(c + d)). We then introduce droplets containing the second species from the bottom flow focusing device (Fig. 5(e)). Here lower flow rates than for the first droplets are used to prevent accidentally knocking any further droplet out

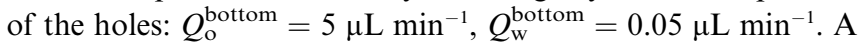
loaded array, with two droplets per trap site, each with different contents, is shown in Fig. 6(a).

Having established the ability to fill the holes with droplets we can then use laser induced droplet merging ${ }^{15}$ to start the chemical reaction. With the control we have over the positioning of the laser this means that we can target a specified droplet pair in the array. The power used is $200 \mathrm{~mW}$, and a dwell time at the droplet interface of $100 \mathrm{~ms}$ is required before any fusion takes place when laser position is well placed.

The process of laser initiated droplet merging is shown in Fig. 6(b) and ESI movie S4, $\dagger$ where we choose to react the droplets such that they form a ' $\mathrm{W}$ ' pattern. The track of the laser is shown by the solid and dashed lines in Fig. 6(b), which correspond to a time $t=117 \mathrm{~ms}$ after the merging of the drops at the top-center location. Fig. 6(c) shows the final state where only

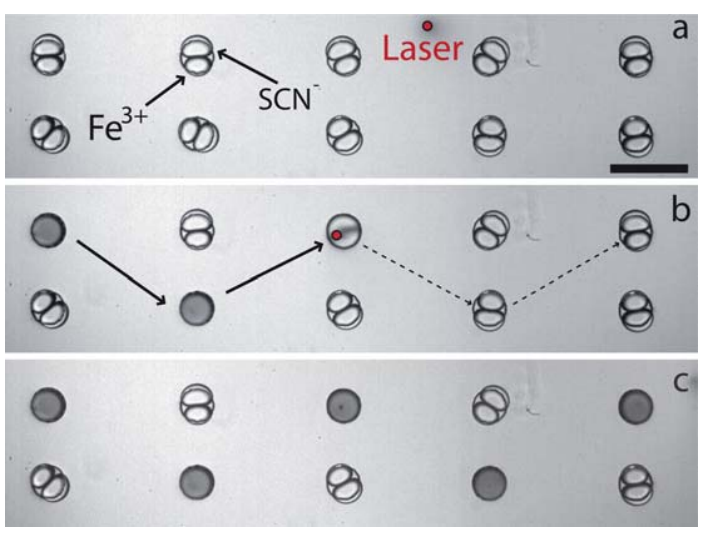

Fig. 6 Laser induced merging of droplets in anchor sites. (a) Filled droplet array. Laser spot is shown as a red dot. (b) The laser is scanned in the pattern shown. As it stops on the droplet-droplet interface it initiates fusion and a chemical reaction takes place. The solid line shows where the beam has been, the dashed line its future path. (c) The laser completes its path leaving 5 anchor points with a reaction product and all other droplets undisturbed. Scale bar is $400 \mu \mathrm{m}$.

the droplets illuminated by the laser have undergone the reaction.

\section{Summary and discussions}

In summary, we have demonstrated the first device that allows a selective patterning of a 2D array with microfluidic droplets. This is performed either through selectively filling or emptying the array, or by triggering a chemical reaction on demand. The approach relies on combining the rails and anchors approach, which provides robust passive manipulation, and the localized heating from the laser which adds selectivity and local actuation.

Both of these approaches are highly scalable, independent of the microchannel material and rely on very simple micro-fabrication: the rails and anchors are produced using lithographic techniques and can easily be densified over wide areas. The design of the test section is nearly common to all of the devices, which only differ in the pattern of rails and anchors that are designed for a specific application. This further simplifies the fabrication in large quantities for commercial applications. As for the optical heating, any number of operations can be performed with single beam by simply scanning the laser focus or by using holographic techniques to split one beam into a complex pattern. ${ }^{22}$ The tight focus of the laser allows manipulation of individual droplets, even in very dense arrays, providing a clear advantage over electrical or acoustic methods.

The throughput of the devices presented here is appropriate for many applications that require a high level of control, since all of the experiments presented here lasted less than a few minutes using purely manual control of the flow rate and the laser position. This duration can be further reduced through use of an automated time-sharing of the laser heating, which can produce many operations simultaneously, or through optimization of the device designs. In the case of the droplet extraction for instance, a different pattern of anchors or the addition of gutter rails would allow the device to function at higher oil velocity, therefore allowing much faster sorting of the drops. 
Increasing the throughput in the case of the array filling depends on the ability to derail rapidly moving drops. This will be realizable by improving the tolerance on the micro-fabrication process, which would allow the distance between the side-rails and the central rail to be better calibrated for the drop sizes. By optimizing this distance, drops can be derailed with a minimal deflection, which would allow us to work at higher flow rates.

Finally, future work will have to investigate the effect of the laser heating on the contents of the droplets, as well as ways to minimize these effects such as pulsing the laser source. While we expect that the heating can damage some proteins or sensitive biological material, it should still allow DNA or chemical manipulation without any significant artifacts.

\section{Acknowledgments}

Special thanks to Estelle Mayot for providing the perfluorinated surfactants used in our studies. The authors also thank Caroline Frot for microfabrication assistance. This project was partially supported by a 'Royal Society International Joint Project'. D. M. is a Royal Society University Research Fellow. The authors finally acknowledge the financial support from CNRS and Ecole Polytechnique.

\section{References}

1 S. K. Cho, H. Moon and C. J. Kim, J. Microelectromech. Syst., 2003, 12, 70-80.

2 A. Wixforth, Superlattices Microstruct., 2003, 33, 389-396.

3 L. Mugherli, O. N. Burchak, L. A. Balakireva, A. Thomas, F. Chatelain and M. Y. Balakirev, Angew. Chem. Int. Ed., 2009, $1433-7851$.
4 H. Kim, S. Vishniakou and G. W. Faris, Lab Chip, 2009, 9, 12301235 .

5 C. N. Baroud, F. Gallaire and R. Dangla, Lab Chip, 2010, 10, 2032 2045.

6 H. Boukellal, S. Selimovic, Y. Jia, G. Cristobal and S. Fraden, Lab Chip, 2009, 9, 331-338.

7 W. Shi, J. Qin, N. Ye and B. Lin, Lab Chip, 2008, 8, 1432-1435.

8 P. Laval, N. Lisai, J. B. Salmon and M. Joanicot, Lab Chip, 2007, 7, $829-834$.

9 C. H. J. Schmitz, A. C. Rowat, S. Koester and D. A. Weitz, Lab Chip, 2009, 9, 44- 49.

10 J. Shim, G. Cristobal, D. R. Link, T. Thorsen, Y. Jia, K. Piattelli and S. Fraden, J. Am. Chem. Soc., 2007, 129, 8825-8835.

11 A. Huebner, D. Bratton, G. Whyte, M. Yang, A. J. deMello, C. Abell and F. Hollfelder, Lab Chip, 2009, 9, 692-698.

12 Y. Bai, X. He, D. Liu, S. N. Patil, D. Bratton, A. Huebner, F. Hollfelder, C. Abell and W. T. S. Huck, Lab Chip, 2010, 10, 1281-1285.

13 A. Huebner, C. Abell, W. T. S. Huck, C. N. Baroud and F. Hollfelder, Anal. Chem., 2011, 83, 1462-1468.

14 P. Abbyad, R. Dangla, A. Alexandrou and C. N. Baroud, Lab Chip, 2011, 11, 813-821.

15 C. N. Baroud, M. R. de Saint Vincent and J. P. Delville, Lab Chip, 2007, 7, 1029-1033.

16 C. N. Baroud, J. P. Delville, F. Gallaire and R. Wunenburger, Phys. Rev. E, 2007, 75, 046302 .

17 M. L. Cordero, D. R. Burnham, C. N. Baroud and D. McGloin, Appl. Phys. Lett., 2008, 93, 034107.

18 K. Stephan, P. Pittet, L. Renaud, P. Kleimann, P. Morin, N. Ouaini and R. Ferrigno, J. Micromech. Microeng., 2007, 17, N69-N74.

19 J. Clausell-Tormos, D. Lieber, J. C. Baret, A. El-Harrak, O. J. Miller, L. Frenz, J. Blouwolff, K. J. Humphry, S. Köster and H. Duan, et al., Chem. Biol., 2008, 15, 427-437.

20 R. Dangla, S. Lee and C. N. Baroud, Phys. Rev. Lett., 2011, 107, 124501.

21 E. Verneuil, M. L. Cordero, F. Gallaire and C. N. Baroud, Langmuir, 2009, 25, 5127-5134.

22 D. G. Grier, Nature, 2003, 424, 810-816. 